\title{
Thickness of preperitoneal fat as a predictor of malignancy in overweight and obese women with endometrial polyps
}

\author{
ANDREA CIAVATTINI $^{1}$, JACOPO DI GIUSEPPE ${ }^{1}$, NICOLÒ CLEMENTE ${ }^{1}$, LORENZO MORICONI $^{1}$, \\ GIOVANNI DELLI CARPINI $^{1}$, NINA MONTIK ${ }^{1}$ and LAURA MAZZANTI ${ }^{2}$ \\ ${ }^{1}$ Gynecological Section, Women's Health Sciences Department; ${ }^{2}$ Biochemistry Section, \\ Department of Clinical Sciences, Polytechnic University of Marche, Ancona I-60123, Italy
}

Received December 29, 2014; Accepted October 27, 2015

DOI: $10.3892 / \mathrm{ol} .2016 .4186$

\begin{abstract}
The aim of the present study was to determine the association between the thickness of preperitoneal fat (PFT), utilized as an indicator of visceral fat deposition, and the risk of premalignant and malignant changes of endometrial polyps (EPs) in overweight and obese women. Overweight and obese women who had undergone diagnostic outpatient hysteroscopy and subsequent endometrial polypectomy between January 2010 and May 2013 were analyzed. Each patient underwent a transabdominal ultrasound for the purpose of measuring the thickness of preperitoneal fat. A total of 146 overweight or obese women were analyzed. The overall incidence of malignant or premalignant lesions was $8.2 \%$. Notably, 5 patients $(3.4 \%)$ received a histopathological diagnosis of complex hyperplasia with atypia, while polyps harboring carcinoma were diagnosed in 7 cases $(4.8 \%)$. A significantly increased PFT was observed in women exhibiting preneoplastic and neoplastic lesions, compared with women with benign EPs (mean $\pm \mathrm{SD}, 23.2 \pm 3.7$ vs. $15.9 \pm 8.3$; $\mathrm{P}<0.01)$. Patient age of $>60$ years was significantly associated with malignant progression of EPs, while body mass index, menopausal status, arterial hypertension, diabetes, abnormal uterine bleeding, hormone replacement therapy and tamoxifen treatment demonstrated no significant association with the development of preneoplastic and neoplastic lesions of the endometrium. In a multivariate analysis, only PFT maintained a significant correlation with the diagnosis of preneoplastic and neoplastic lesions on EPs (odds ratio, 1.14; 95\% confidence interval, 1.04-1.26). Ultrasound evaluation of PFT in overweight and obese women may be useful for the identification of a particularly high-risk subgroup of women. Therefore, regardless of symptoms or additional clinical
\end{abstract}

Correspondence to: Professor Andrea Ciavattini, Gynecological Section, Woman's Health Sciences Department, Polytechnic University of Marche, 11 Via Corridoni, Ancona I-60123, Italy

E-mail: ciavattini.a@libero.it

Key words: endometrial polyps, preperitoneal fat thickness, malignant progression variables, these particularly high-risk women require appropriate counseling and prompt surgical removal of EPs.

\section{Introduction}

In recent years, the use of routine ultrasound evaluation of the uterine cavity and diagnostic outpatient hysteroscopy has increased the diagnosis of endometrial polyps (EPs) and endometrial cancer precursors (1). Though EPs are largely described as benign lesions, a number of studies have reported that EPs and endometrial carcinoma may coexist in one patient, and that premalignant changes occasionally present in EPs are identical to those observed in atypical endometrial hyperplasia (2,3). Several studies have described the risk of endometrial cancer in women with EPs, and have reported conflicting data; however, the majority of studies have reported an increased risk of malignancy in older women (>60 years of age) exhibiting abnormal uterine bleeding (AUB) (4-7).

The association between obesity, visceral adiposity and risk of endometrial cancer in patients with EPs is currently under debate.

Obesity is a major contributor to the global burden of disability and chronic disease, and is a significant public health concern (8). It has been estimated that $\sim 20 \%$ of all cancer cases are caused by excess weight (9). In particular, endometrial cancer demonstrates an association with an increased body mass index (BMI), with $39 \%$ of cases occurring in obese women (10). Several mechanisms may promote endometrial cancer occurrence or progression in overweight or obese women. Primarily, this association may be explained by an increase in circulating estrogens that arise from the aromatization of androgenic precursors in adipose tissue, leading to endometrial cell proliferation (11). In addition, mechanisms involving insulin resistance and hyperinsulinemia, insulin growth factors (IGFs) or adipose-derived adipokines (such as leptin and adiponectin) appear to be involved in carcinogenesis (12). Furthermore, the chronic inflammatory process associated with visceral adiposity, which may be confirmed by detection of increased levels of C-reactive protein (CRP), interleukin (IL) 6 , IL8 and tumor necrosis factor $\alpha(\mathrm{TNF} \alpha)$ in the systemic circulation of obese women, may play a role in the development of endometrial cancer (12). 
Though obesity appears to be clearly associated with endometrial cancer development, weight and BMI are not representative of all types of body adipose tissue distribution, and a specific increase in visceral fat, rather than obesity itself, appears to be associated with carcinogenesis (13).

To allow an accurate evaluation of adipose tissue distribution, the utilization of a variety of imaging methods has been proposed. Computed tomography (CT) and magnetic resonance imaging (MRI) are considered to be the most reliable methods for the assessment of adipose tissue distribution (14). However, due to the high cost of CT and MRI, ultrasound has been increasingly employed for evaluation of visceral adipose tissue distribution, via measurement of the thickness of preperitoneal fat (PFT).

PFT is defined as the thickness of the fat tissue between the liver surface and the linea alba, and may be utilized as an indicator of visceral fat deposition (15). PFT may be easily evaluated using transabdominal ultrasound and, due to its potential correlation with the development of endometrial cancer, it may be useful as a predictor for potential malignancy of endometrial lesions (13).

The aim of the present study was to determine the association between the PFT and the risk of premalignant and malignant changes in EPs in a high-risk population of overweight and obese women, taking into account potential clinical and demographic confounders.

\section{Materials and methods}

Patients and study design. The medical records of overweight (BMI, 25.0-29.9 kg/m²), moderately obese (BMI, $30.0-34.9 \mathrm{~kg} / \mathrm{m}^{2}$ ) and severely obese women (BMI, $\geq 35 \mathrm{~kg} / \mathrm{m}^{2}$ ), who had undergone routine transvaginal ultrasound and subsequent diagnostic outpatient hysteroscopy and endometrial polypectomy at the Woman's Health Sciences Department, Gynecological Section, Polytechnic University of Marche (Ancona, Italy), between January 2010 and May 2013, were retrospectively analyzed in an observational cohort study. The approval of the Polytechnic University of Marche ethical committee was obtained for the routine collection of data.

All patients underwent a complete clinical evaluation, with a physical examination, transabdominal and transvaginal ultrasound and diagnostic outpatient hysteroscopy, prior to undergoing endometrial polypectomy. Written informed consent was obtained from each patient prior to the procedure.

PFT measurements. Transabdominal ultrasounds were performed with the purpose of measuring the PFT, defined as the thickness of the fat tissue between the liver surface and the linea alba, and utilized as an indicator of visceral fat deposition (15). Ultrasound was performed with a high-resolution ultrasonographic system (Voluson 730 Pro; GE Healthcare Bio-Sciences, Pittsburgh, PA, USA). As previously described by Suzuki et al (15), the PFT was evaluated with the patient in the supine position, and three independent measurements were performed for each patient. The maximum thickness of the PFT was measured using longitudinal scanning, with a 1-5 MHz convex probe (4C-A H46701AA; GE Healthcare Bio-Sciences) held perpendicular to the skin at the epigastrium, and scanning was performed along the abdominal median line from the xiphoid process to the umbilicus at the anterior surface of the liver. All images were captured immediately following a breath to avoid the influence of abdominal wall tension or respiratory status.

Diagnostic hysteroscopy. Patients in whom endometrial lesions were detected during the transvaginal ultrasound underwent a diagnostic outpatient hysteroscopy. A Storz endoscope (KARL STORZ GmbH \& Co. KG, Tuttlingen, Germany) with a 5-mm diagnostic sheath was used, with saline solution as a distention medium. The vaginoscopic approach was used in all cases to avoid patient discomfort or pain not directly related to uterine examination. Neither analgesia nor local anesthesia were administered during the procedures.

Clinical and demographic patient characteristics. The clinical and demographic characteristics of each patient were recorded. In particular, data regarding arterial hypertension (defined as diastolic pressure $\geq 90 \mathrm{mmHg}$ and/or systolic pressure $\geq 140 \mathrm{mmHg}$ ), BMI, diabetes mellitus (fasting glucose, $\geq 126 \mathrm{mg} / \mathrm{dl}$ ), AUB, hormonal and tamoxifen therapy, and number of pregnancies were recorded. Patients were considered to be postmenopausal following a period of $\geq 12$ months of amenorrhea. AUB was defined as any vaginal bleeding in postmenopausal women not receiving hormone replacement therapy (HRT), or in premenopausal women exhibiting irregular bleeding. Women in treatment with tamoxifen as an adjuvant therapy for breast cancer were additionally included in the study group. Women with cervical cancer, complex adnexal pathology or severe liver pathology, or who were pregnant, were excluded from the study.

Surgical management. The hysteroscopic polypectomies were performed by a senior gynecological surgeon under general anesthesia. Procedures were performed using a $10-\mathrm{mm}$ resectoscope with $0^{\circ}$ forward lens and a $2.5 \mathrm{~mm}$ Versapoint electrode (Gynecare; Johnson \& Johnson, New Brunswick, NJ, USA), with the use of saline solution as the distension media. The aim of the resection was the complete removal of the EPs, and evaluation was performed of the endocervical canal, endometrial surface, vascularity, tubal ostia and synechiae. No intraoperative or postoperative complications were recorded in any of the patients. In premenopausal women, the procedure was conducted during the proliferative phase of the menstrual cycle. Excised specimens were sent to the Institute of Pathological Anatomy of the Reunited Hospitals, Polytechnic University of Marche (Ancona, Italy) for histopathological examination. Patients with a diagnosis of submucosal leiomyoma or uterine adenomyoma were excluded from the study.

Histological diagnosis distinguished between benign EPs, premalignant EPs (complex hyperplasia with atypia) and polyps harboring carcinoma. The histopathological definitions of EP, hyperplasia and adenocarcinoma have been reported in a previous study (16).

Statistical analysis. Statistical analysis was performed using SPSS version 22.0 (IBM SPSS, Armonk, NY, USA). The Student's $t$-test, $\chi^{2}$ test and Fisher's exact test were used to 
Table I. Clinical and demographic characteristics of the study cohort.

\begin{tabular}{lcccr}
\hline Variable & $\begin{array}{c}\text { All patients } \\
(\mathrm{n}=146)\end{array}$ & $\begin{array}{c}\text { Benign polyps } \\
(\mathrm{n}=134)\end{array}$ & $\begin{array}{c}\text { Preneoplastic and } \\
\text { neoplastic lesions (n=12) }\end{array}$ & P-value \\
\hline Age, years (mean $\pm \mathrm{SD})$ & $55.8 \pm 13.4$ & $54.9 \pm 13.1$ & $65.9 \pm 12.9$ & 0.006 \\
$<40, \mathrm{n}(\%)$ & $17(11.7)$ & $16(11.9)$ & $1(8.3)$ & 0.922 \\
$40-50, \mathrm{n}(\%)$ & $37(25.3)$ & $36(26.9)$ & $1(8.3)$ & 0.283 \\
$50-60, \mathrm{n}(\%)$ & $38(26.0)$ & $37(27.6)$ & $9(8.3)$ & 0.264 \\
$>60, \mathrm{n}(\%)$ & $54(37.0)$ & $45(33.6)$ & $30.2 \pm 3.5$ & 0.011 \\
BMI, kg/m $($ mean $\pm \mathrm{SD})$ & $29.5 \pm 4.5$ & $29.4 \pm 4.6$ & $7(58.3)$ & 0.558 \\
$25-29.9, \mathrm{n}(\%)$ & $95(65.1)$ & $88(65.7)$ & $3(25.0)$ & 0.842 \\
$30-34.9, \mathrm{n}(\%)$ & $37(25.3)$ & $34(25.4)$ & $2(16.7)$ & 0.752 \\
$\geq 35, \mathrm{n}(\%)$ & $14(9.6)$ & $12(9.0)$ & $5(83.3)$ & 0.722 \\
Menopause, $\mathrm{n}(\%)$ & $89(61.0)$ & $79(59.0)$ & $4(41.6)$ & 0.178 \\
Abnormal uterine bleeding, $\mathrm{n}(\%)$ & $61(41.8)$ & $56(41.8)$ & $0(0.0)$ & 0.770 \\
Nulliparity, $\mathrm{n}(\%)$ & $26(17.8)$ & $22(16.4)$ & $6(50.0)$ & 0.283 \\
Diabetes mellitus, $\mathrm{n}(\%)$ & $5(3.4)$ & $5(3.7)$ & $1(8.3)$ & 0.877 \\
Hypertension, $\mathrm{n}(\%)$ & $60(41.1)$ & $54(40.3)$ & $0(0.0)$ & 0.727 \\
Breast cancer, $\mathrm{n}(\%)$ & $3(2.1)$ & $2(1.5)$ & $0(0.0)$ & 0.597 \\
Tamoxifen, $\mathrm{n}(\%)$ & $4(2.7)$ & $4(3.0)$ & $23.2 \pm 3.7$ & 0.754 \\
Hormone replacement therapy, $\mathrm{n}(\%)$ & $8(5.5)$ & $8(6.0)$ & & 0.831 \\
PFT, mm (mean \pm SD) & $16.5 \pm 8.2$ & $15.9 \pm 8.3$ & 0.003 \\
\hline
\end{tabular}

BMI, body mass index; PFT, preperitoneal fat thickness; SD, standard deviation.

analyze categorical or continuous variables, as appropriate. $\mathrm{P}<0.05$ was considered to indicate a statistically significant difference. Associations were expressed with $95 \%$ confidence intervals (CIs). Multivariate logistic regression was used to adjust for confounding factors identified through the results of univariate and stratified analyses. Analysis of the area under the receiver operating characteristic curve was used as a post-hoc test.

\section{Results}

Clinical and demographic patient characteristics. A total of 146 overweight or obese women fulfilling the study inclusion criteria were considered in the present study. Following endometrial polypectomy, the overall incidence of malignant or premalignant lesions was $8.2 \%$ : 5 patients $(3.4 \%)$ received a histopathological diagnosis of premalignant polyps, while polyps harboring carcinoma were diagnosed in 7 cases $(4.8 \%)$. The mean patient age was $55.8 \pm 13.4$ years (range, $27-87$ years), and 89 women $(61.0 \%)$ had postmenopausal status. The mean BMI was $29.5 \pm 4.5 \mathrm{~kg} / \mathrm{m}^{2}$ (range, 25.0-58.6). A total of 95 patients $(65.1 \%)$ were overweight, 37 patients $(25.3 \%)$ were moderately obese and $14(9.6 \%)$ were severely obese. The primary demographic and clinical characteristics of the study cohort and the histopathological findings following endometrial polypectomy are shown in Table I.

Univariate analysis. Age was significantly associated with the development of preneoplastic and neoplastic lesions of the
Table II. Multivariate logistic regression.

\begin{tabular}{lccr}
\hline Variable & Odds ratio & $95 \%$ CI & P-value \\
\hline Age, years & 1.06 & $0.96-1.16$ & 0.247 \\
Age $>$ 60 years & 2.00 & $0.19-20.82$ & 0.615 \\
PFT & 1.14 & $1.04-1.26$ & 0.006
\end{tabular}

PFT, preperitoneal fat thickness; CI, confidence interval.

endometrium, and patients $>60$ years old had a significantly increased risk of malignancy (75.0 vs. $33.6 \%$; $\mathrm{P}=0.011)$. By contrast, BMI, menopausal status, AUB, arterial hypertension, diabetes, HRT, tamoxifen treatment and number of pregnancies demonstrated no significant association with the development of preneoplastic and neoplastic lesions of the endometrium.

No significant difference was observed between the rate of preneoplastic and neoplastic lesions on EPs in overweight, moderately obese and severely obese women.

Multivariate analysis. A significantly increased PFT was observed in women exhibiting preneoplastic and neoplastic lesions, compared with women with benign EPs (23.2 \pm 3.7 vs. $15.9 \pm 8.3 \mathrm{~mm} ; \mathrm{P}=0.003)$. The mean PFT for the entire study population was $16.5 \pm 8.2 \mathrm{~mm}$ (range, 6.7-41.6 $\mathrm{mm}$ ); in the 12 cases with histopathologically diagnosed preneoplastic and neoplastic lesions in excised EPs, the mean PFT was 
$23.2 \pm 3.7 \mathrm{~mm}$, while in the remaining 134 cases with benign EPs, the mean PFT was $15.9 \pm 8.3 \mathrm{~mm}$.

Significant variables obtained by univariate analysis were included in the multiple regression model. Among these variables, only PFT maintained a significant correlation with a diagnosis of preneoplastic and neoplastic lesions in EPs (odds ratio, 1.14; 95\% CI, 1.04-1.26; $\mathrm{P}=0.006$; Table II).

\section{Discussion}

EPs are frequently described as benign lesions; however, a $0.3-4.8 \%$ risk of malignant progression has been reported $(2,5,17-20)$. In the present patient cohort, the rate of malignant and premalignant lesions in excised EPs was $8.2 \%$. This appeared to be increased compared with the rates reported in previous studies; however, this may be due to the fact that analysis was restricted to a specific high-risk population of overweight and obese women. The presence of premalignant and malignant alterations in $>8 \%$ of the patients with EPs is interesting, and it may be useful for the identification of specific clinical characteristics that are associated with the risk of malignant progression of EPs, which could potentially allow a 'very high-risk' population of women to be identified.

Obesity is considered to be a significant risk factor for the development of endometrial cancer $(9,10)$. Furthermore, it is considered to be a risk factor for the development of EPs themselves (21), and in particular appears to lead to malignant progression (22-24). In a study by Litta et al (25) a linear correlation between BMI and risk of malignancy in women with EPs was observed.

Obesity is a significant contributor to the global burden of chronic illnesses, including cancer development (8-10). In European countries, the prevalence of obesity varies from 6 to $20 \%$, with increased prevalence in Central and Eastern European countries, and reduced rates in France, Italy and certain Scandinavian countries (26). In the United States, the prevalence of obesity has increased from $15 \%$ in the early 1970 s to the most recent estimate of $34 \%$ in $2009-2010(27,28)$.

As previously stated, obesity appears to have a significant role in the development of endometrial cancer. However, weight and BMI are not representative of all types of body adipose tissue distribution, and an increase in visceral fat, rather than obesity itself, appears to be associated with carcinogenesis. A visceral fat area $>100 \mathrm{~cm}^{2}$ at the umbilical level is considered to be a risk factor for cardiac disorders, diabetes mellitus and various types of cancer, and is defined as 'visceral obesity' (29). However, among overweight and obese women, only $66 \%$ possess a visceral fat area $>100 \mathrm{~cm}^{2}$ (30). Notably, the prevalence of complications is increased in patients with a visceral fat area $>100 \mathrm{~cm}^{2}$, even if these patients are not obese (31). The results of the present study suggest that weight and BMI alone do not assist with exact quantification of visceral fat.

Increased visceral fat has been associated with increased levels of circulating estrogens in postmenopausal women, reduced progesterone levels in premenopausal women and reduced levels of sex hormone-binding globulin, a protein that binds and modulates the biological activity of estrogens (32). In addition, visceral obesity is associated with decreased glucose tolerance, chronic hyperinsulinemia and increased levels of IGF-1 (33), and these mechanisms have been observed to be involved in endometrial carcinogenesis in type I and type II tumors (11,34). Furthermore, the production of proinflammatory cytokines (TNF $\alpha$, IL6 and CRP) has a significant role in the development of endometrial cancer; however, the underlying mechanisms remain to be elucidated (35). Visceral obesity appears to be associated with chronic inflammatory processes, which may be confirmed by the detection of increased levels of inflammatory markers in the systemic circulation of obese patients (36). Additionally, abdominal fat deposition appears to be associated with reduced levels of adiponectin (33), and it has been proven that adiponectin plays a protective role in carcinogenesis by enhancing insulin sensitivity, and its circulating levels are inversely correlated with cancer occurrence and stage (12).

As previously stated, an increase in visceral fat, rather than obesity itself, may represent a potential risk factor for endometrial cancer and malignant progression in women with EPs. In this context, it is important to find an easy-to-perform, rapid and non-invasive method for the evaluation of visceral adiposity; ultrasound evaluation of PFT may be utilized as an effective indicator of visceral fat deposition. PFT may be easily evaluated with the use of transabdominal ultrasound and, due to its potential correlation with the development of endometrial cancer, it may be useful, particularly in high-risk overweight or obese women, as a predictor of malignancy in endometrial lesions (13).

The results of the present study revealed a significantly increased PFT value in overweight and obese women diagnosed with preneoplastic and neoplastic EPs. In addition, this association persisted in a multiple regression analysis. By contrast, BMI demonstrated no significant association with the development of preneoplastic and neoplastic lesions of the endometrium.

The present study, which was consistent with previously published data by Baiocchi et al (16), did not demonstrate any statistically significant difference between asymptomatic and symptomatic patients with regard to the presence of benign polyps and preneoplastic or neoplastic lesions. A potential explanation for this may be associated with the early diagnosis of polyps using transvaginal ultrasound, which is able to detect small polyps and endometrial thickness before they begin to bleed.

In previously published studies, advanced age ( $>60$ years), menopausal status, HRT and AUB were observed to be risk factors for malignancy in women with EPs $(4,7,16-18,37)$. In the present study, an age of $>60$ years was significantly associated with the risk of malignant progression of EPs; however, none of the remaining clinical variables considered were found to demonstrate such an association. These findings may be due to the restricted high-risk population considered in the present study.

In contrast to the results of previous studies $(38,39)$, the present study did not identify HRT and tamoxifen treatment to be significant predictors of endometrial cancer. This discrepancy may potentially be attributed to the small number of women included in the present study that were actually treated with HRT or tamoxifen.

In conclusion, ultrasound evaluation of PFT in overweight and obese women may assist with the identification of a 
particularly high-risk subgroup of women. Therefore, regardless of symptoms or other clinical variables, these particularly high-risk women require appropriate counseling and prompt surgical removal of EPs. The small number of cases investigated in the present study did not allow the definition of an ideal threshold for PFT with regard to risk of malignancy in EPs. However, the identification of a cut-off level of PFT for the risk of malignant progression of EPs in obese women is advisable, and further studies may be required to achieve this.

\section{References}

1. Litta P, Merlin F, Saccardi C, Pozzan C, et al: Role of hysteroscopy with endometrial biopsy to rule out endometrial cancer in postmenopausal women with abnormal uterine bleeding. Maturitas 50: 117-123, 2005.

2. Ferrazzi E, Zupi E, Leone FP, Savelli L, Omodei U, Moscarini M, Barbieri M, Cammareri G, Capobianco G, Cicinelli E, et al: How often are endometrial polyps malignant in asymptomatic postmenopausal women? A multicenter study. Am J Obstet Gynecol 200: 235.e1-e6, 2009.

3. Litta P, Bartolucci C, Saccardi C, Codroma A, Fabris A, Borgato S and Conte L: Atypical endometrial lesions: Hysteroscopic resection as an alternative to hysterectomy. Eur J Gynaecol Oncol 34: 51-53, 2013

4. Costa-Paiva L, Godoy CE Jr, Antunes A Jr, Caseiro JD, Arthuso M and Pinto-Neto AM: Risk of malignancy in endometrial polyps in premenopausal and postmenopausal women according to clinicopathologic characteristics. Menopause 18: 1278-1282, 2011.

5. Wethington SL, Herzog TJ, Burke WM, Sun X, Lerner JP, Lewin SN and Wright JD: Risk and predictors of malignancy in women with endometrial polyps. Ann Surg Oncol 18: 3819-3823, 2011

6. Machtinger R, Korach J, Padoa A, Fridman E, Zolti M, Segal J, Yefet Y, Goldenberg M and Ben-Baruch G: Transvaginal ultrasound and diagnostic hysteroscopy as a predictor of endometrial polyps: Risk factors for premalignancy and malignancy. Int J Gynecol Cancer 15: 325-328, 2005.

7. Antunes A Jr, Costa-Paiva L, Arthuso M, Costa JV and Pinto-Neto AM: Endometrial polyps in pre- and postmenopausa women: Factors associated with malignancy. Maturitas 57: 415-421, 2007.

8. Swinburn BA, Sacks G, Hall KD, McPherson K, Finegood DT, Moodie ML and Gortmaker SL: The global obesity pandemic: Shaped by global drivers and local environments. Lancet 378 804-814, 2011.

9. Wolin KY, Carson K and Colditz GA: Obesity and cancer Oncologist 15: 556-565, 2010.

10. Renehan AG, Tyson M, Egger M, Heller RF and Zwahlen M: Body-mass index and incidence of cancer: A systematic review and meta-analysis of prospective observational studies. Lancet 371 569-578, 2008.

11. Calle EE and Kaaks R: Overweight, obesity and cancer: Epidemiological evidence and proposed mechanisms. Nat Rev Cancer 4: 579-591, 2004

12. De Pergola G and Silvestris F: Obesity as a major risk factor for cancer. J Obes 2013: 291546, 2013.

13. Friedenreich C, Cust A, Lahmann PH, Steindorf K, Boutron-Ruault MC, Clavel-Chapelon F, Mesrine S, Linseisen J, Rohrmann S, Boeing $\mathrm{H}$, et al: Anthropometric factors and risk of endometrial cancer: The European prospective investigation into cancer and nutrition. Cancer Causes Control 18: 399-413, 2007.

14. Val-Laillet D, Blat S, Louveau I and Malbert CH: A computed tomography scan application to evaluate adiposity in a minipig model of human obesity. Br J Nutr 104: 1719-1728, 2010.

15. Suzuki R, Watanabe S, Hirai Y, Akiyama K, Nishide T, Matsushima Y, Murayama H, Ohshima H, Shinomiya M, Shirai K, et al: Abdominal wall fat index, estimated by ultrasonography, for assessment of the ratio of visceral fat to subcutaneous fat in the abdomen. Am J Med 95: 309-314, 1993.

16. Baiocchi G, Manci N, Pazzaglia M, Giannone L, Burnelli L, Giannone E, Fratini D and Di Renzo GC: Malignancy in endometrial polyps: A 12-year experience. Am J Obstet Gynecol 201: 462.e1-4, 2009
17. Savelli L, De Iaco P, Santini D, Rosati F, Ghi T, Pignotti E and Bovicelli L: Histopathologic features and risk factors for benignity, hyperplasia, and cancer in endometrial polyps. Am J Obstet Gynecol 188: 927-931, 2003

18. Lee SC, Kaunitz AM, Sanchez-Ramos L and Rhatigan RM: The oncogenic potential of endometrial polyps: A systematic review and meta-analysis. Obstet Gynecol 116: 1197-1205, 2010.

19. Bettocchi S, Ceci O, Di Venere R, Pansini MV, Pellegrino A, Marello F and Nappi L: Advanced operative office hysteroscopy without anaesthesia: Analysis of 501 cases treated with a $5 \mathrm{Fr}$. bipolar electrode. Hum Reprod 17: 2435-2438, 2002.

20. Wang JH, Zhao J and Lin J: Opportunities and risk factors for premalignant and malignant transformation of endometrial polyps: Management strategies. J Minim Invasive Gynecol 17: 53-58, 2010.

21. Serhat E, Cogendez E, Selcuk S, Asoglu MR, Arioglu PF and Eren S: Is there a relationship between endometrial polyps and obesity, diabetes mellitus, hypertension? Arch Gynecol Obstet 290: 937-941, 2014.

22. Ward KK, Roncancio AM, Shah NR, Davis MA, Saenz CC, McHale MT and Plaxe SC: The risk of uterine malignancy is linearly associated with body mass index in a cohort of US women. Am J Obstet Gynecol 209: 579.e1-5, 2013.

23. Acmaz G, Aksoy H, Albayrak E, Baser M, Ozyurt S, Aksoy U and Unal D: Evaluation of endometrial precancerous lesions in postmenopausal obese women - a high risk group? Asian Pac J Cancer Prev 15: 195-198, 2014.

24. Gregoriou O, Konidaris S, Vrachnis N, Bakalianou K, Salakos N, Papadias K, Kondi-Pafiti A and Creatsas G: Clinical parameters linked with malignancy in endometrial polyps. Climacteric 12 : 454-458, 2009.

25. Litta P, Di Giuseppe J, Moriconi L, Delli Carpini G, Piermartiri MG and Ciavattini A: Predictors of malignancy in endometrial polyps: A multi-institutional cohort study. Eur J Gynaecol Oncol 35 382-386, 2014.

26. Rabin BA, Boehmer TK and Brownson RC: Cross-national comparison of environmental and policy correlates of obesity in Europe. Eur J Public Health 17: 53-61, 2007.

27. Freedman DS; Centers for Disease Control and Prevention (CDC) Obesity - United States, 1988-2008. MMWR Surveill Summ 60 (Suppl): 73-77, 2011.

28. Ogden CL, Carroll MD, Kit BK and Flegal KM: Prevalence of obesity in the United States, 2009-2010. NCHS Data Brief 82: 1-8, 2012.

29. No authors listed: Obesity: Preventing and managing the global epidemic. Report of a WHO consultation. World Health Organ Tech Rep Ser 894: i-xii, 1-253, 2000

30. Koda M, Senda M, Kamba M, Kimura K and Murawaki Y: Sonographic subcutaneous and visceral fat indices represent the distribution of body fat volume. Abdom Imaging 32: 387-392, 2007.

31. Bouchard C, Deprés JP and Tremblay A: Exercise and obesity. Obes Res 1: 133-147, 1993

32. Bal Y, Adas M and Helvaci A: Evaluation of the relationship between insulin resistance and plasma tumor necrosis factor-alpha, interleukin-6 and C-reactive protein levels in obese women. Bratisl Lek Listy 111: 200-204, 2010.

33. Milewicz A, Jedrzejuk D, Dunajska K and Lwow F: Waist circumference and serum adiponectin levels in obese and non-obese postmenopausal women. Maturitas 65: 272-275, 2010.

34. McTiernan A, Irwin M and Vongruenigen V: Weight, physical activity, diet, and prognosis in breast and gynecologic cancers. J Clin Oncol 28: 4074-4080, 2010.

35. Ciortea R, Mihu D, Costin N, Fufezan O, Feier D, Coman A and Bondor C: Utility of the ultrasound evaluation of intraperitoneal fat in correlation with endometrial cancer. Appl Med Inform 28: 37-44, 2011.

36. Soliman PT, Wu D, Tortolero-Luna G, Schmeler KM, Slomovitz BM, Bray MS, Gershenson DM and Lu KH: Association between adiponectin, insulin resistance, and endometrial cancer. Cancer 106: 2376-2381, 2006.

37. Giordano G, Gnetti L, Merisio C and Melpignano M: Postmenopausal status, hypertension and obesity as risk factors for malignant transformation in endometrial polyps. Maturitas 56: 190-197, 2007.

38. Oguz S, Sargin A, Kelekci S, Aytan H, Tapisiz OL and Mollamahmutoglu L: The role of hormone replacement therapy in endometrial polyp formation. Maturitas 50: 231-236, 2005.

39. Bergman L, Beelen ML, Gallee MP, Hollema H, Benraadt J and van Leeuwen FE: Risk and prognosis of endometrial cancer after tamoxifen for breast cancer. Comprehensive cancer centres ALERT group. Assessment of liver and endometrial cancer risk following tamoxifen. Lancet 356: 881-887, 2000 . 